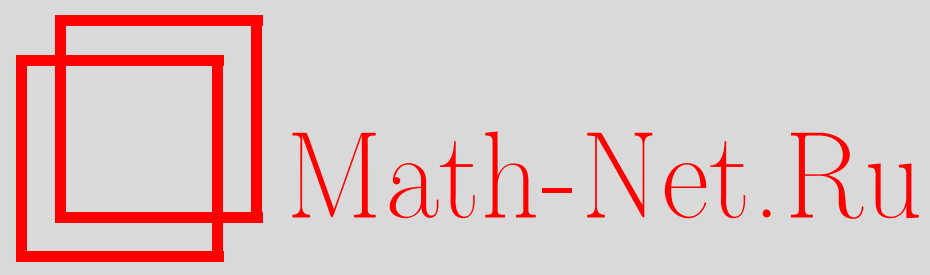

Г. И. Лаптев, Возрастающие монотонные операторы в банаховом пространстве, Матем. заметки, 2002, том 71, выпуск 2, 214-226

DOI: https://doi.org/10.4213/mzm340

Использование Общероссийского математического портала Math-Net.Ru подразумевает, что вы прочитали и согласны с пользовательским соглашением http://www.mathnet.ru/rus/agreement

Параметры загрузки:

IP : 3.85 .5 .30

26 апреля 2023 г., 14:57:15 


\section{ВОЗРАСТАЮЩИЕ МОНОТОННЫЕ ОПЕРАТОРЫ В БАНАХОВОМ ПРОСТРАНСТВЕ}

\section{Г.И. Лаптев}

Оператор $A$, действующий из сепарабельного рефиексивного банахова пространства $X$ в его сопряженное $X^{\prime}$, назван возрастающим, если $\|A u\| \rightarrow \infty$, когда $\|u\| \rightarrow \infty$. Найдены необходимые и достаточные условия возрастания операторов суперпозиции. Анализируется связь свойств возрастания и коэрцитивности для дифференциальных операторов в частных производных монотонного типа. Указьваются дополнительные условия, при которых уравнение $A u=f$ с возрастающим оператором $A$ допускает решение.

Библиограффия: 8 названий.

Введение. Основная теорема о монотонных операторах в банаховых пространствах утверждает следующее [1, с. 95], [2, гл. 2, § 2, теорема 2.1]. Пусть $X$ - вещественное сепарабельное рефлексивное банахово пространство с сопряженным $X^{\prime}$, и пусть оператор $A: X \rightarrow X^{\prime}$ является радиально непрерывным, монотонньг и коэрцитивным. Тогда уравнение $A u=f$ имеет решение $u \in X$ для каждого элемента $f \in X^{\prime}$. Предпринимались многочисленные попытки ослабить хотя бы одно из приведенных условий на оператор $A$. Монотонность удалось заменить на так назьваемое $(M)$-свойство, которое, видимо, можно считать предельным в рамках теории монотонности. Коэрцитивность часто заменяют на специальное условие роста оператора: $\|A u\| \rightarrow \infty$, если $\|u\| \rightarrow \infty$. С делано это для нечетных операторов. В результате теория разделилась на два параллельных направления. В первом изучаются уравнения с коэрцитивньми операторами, обладающими $(M)$-свойством. При этом преимушественно применяется метод Галёркина. Во втором направлении решаются уравнения с нечетными операторами, удовлетворяющими приведенному вьше условию роста. При этом привлекается теория степени отображения. Полученные в обоих направлениях результаты в деталях изложены в обзорных работах [1]-[5]. В предлагаемой статье изучается записанное вьше условие роста оператора в приложении как к конкретным операторам суперпозиции и к дифференциальным операторам, так и к абстрактньм монотонньм операторам.

В дальнейшем предполагается, что пространства $X$ и $X^{\prime}$ сопряжены относительно двойственности $(f, u)$, где $f \in X^{\prime}, u \in X$. Норма каждого пространства будет сопровождаться его индексом. Например, $\|u\|_{X}$ обозначает норму в пространстве $X$. Оператор $A: X \rightarrow X^{\prime}$ назьвается монотонным, если для всех $u, v \in X$ выполняется соотношение $(A u-A v, u-v) \geqslant 0$. Чаше всего свойство монотонности дополняется условием

Работа выполнена при поддержке Российского фонда фундаментальных исследований, гранты № 01-01-00884 и № 99-01-00045. 
коэрцитивности, которое имеет вид $(A u, u) \geqslant \varphi\left(\|u\|_{X}\right)\|u\|_{X}$, где функция $\varphi(r) \rightarrow \infty$ при $r \rightarrow \infty$. Заметим, что из коэрцитивности вытекает следующее свойство:

$$
\|A u\|_{X^{\prime}} \rightarrow \infty \quad\left(\|u\|_{X} \rightarrow \infty\right)
$$

Соотношениям (0.1) присвоим специальньй термин, взятьй из теории возрастающих функционалов [6, гл. VI, $§ 1]$.

ОПРЕДЕЛЕНИЕ 0.1. Оператор $A: X \rightarrow X^{\prime}$ называется возрастающим, если для него выполняются соотношения $(0.1)$.

Раскроем определение более детально. Введем функцию $m(r)=\inf \|A u\|_{X^{\prime}}$, где функции $u \in X$ пробегают множество $\|u\|_{X}=r$. Согласно определению 0.1 оператор $A$ назьвается возрастающим, если $m(r) \rightarrow \infty$ при $r \rightarrow \infty$.

Напомним еще, что оператор $A: X \rightarrow X^{\prime}$ назьвается положительно однородным порядка $k>0$, если $A(t k)=t^{k} A u$ для любых $u \in X, t>0$. Такие операторы тесно связаны с возрастаюшими операторами. Действительно, пусть положительно однородный порядка $k>0$ оператор $A: X \rightarrow X^{\prime}$ удовлетворяет условию inf $\left\|A u_{0}\right\|_{X^{\prime}}=m_{0}>0$, где точная нижняя грань берется по всем элементам $u_{0} \in X$, для которых $\left\|u_{0}\right\|_{X}=1$. Произвольньй элемент $u \in X$ представим в виде $u=\|u\|_{X} u_{0}$, где $\left\|u_{0}\right\|_{X}=1$. Тогда

$$
\|A u\|_{X^{\prime}}=\left\|A\left(\|u\|_{X} u_{0}\right)\right\|_{X^{\prime}}=\|u\|_{X}^{k}\left\|A u_{0}\right\|_{X^{\prime}} \geqslant\|u\|_{X}^{k} m_{0},
$$

откуда очевидно, что оператор $A: X \rightarrow X^{\prime}$ является возрастаюшим.

1. Возрастающие операторы суперпозиции. Операторы суперпозиции представляют редкий пример нелинейных операторов, для которых многие утверждения могут быть высказаны в терминах необходимых и достаточных условий. Здесь будут указаны необходимые и достаточные условия того, что оператор суперпозиции является возрастающим в пространстве суммируемых функций. Опишем рассматриваемые далее операторы. Пусть задано конечномерное пространство $\mathbb{R}^{m}$ и отображение $a(\xi): \mathbb{R}^{m} \rightarrow \mathbb{R}^{m}$, зависящее от параметра $\xi \in \mathbb{R}^{m}$. Функция $a(\xi)$ предполагается непрерьвной. Пусть $\Omega$ - ограниченная область пространства $\mathbb{R}^{n}$. Введем пространство $\left(L^{p}(\Omega)\right)^{m}$ вектор-функций $\varphi(x)=\left(\varphi_{1}(x), \ldots, \varphi_{m}(x)\right)$ таких, что $\varphi_{k}(x) \in L^{p}(\Omega), k=1, \ldots, m$. Оператор суперпозиции определяется как композиция $A \varphi \equiv a[\varphi(x)]$, которая задает вектор-функцию, определенную для почти всех $x \in \Omega$. Хорошо известно [6, гл. $1, \S 2$, теорема 2.3], что оператор $A:\left(L^{p}(\Omega)\right)^{m} \rightarrow\left(L^{p^{\prime}}(\Omega)\right)^{m}$, где $p+p^{\prime}=p p^{\prime}, p>1$, непрерьвен и ограничен тогда и только тогда, когда

$$
|a(\xi)| \leqslant c_{1}|\xi|^{p-1}+c_{2} \quad\left(c_{1}>0\right) .
$$

Следующее утверждение дает аналогичньй критерий возрастания оператора $A$.

Tеорема 1.1. Oператор суперпозииии $A:\left(L^{p}(\Omega)\right)^{m} \rightarrow\left(L^{p^{\prime}}(\Omega)\right)^{m}$ является непрерывным, ограниченным и возрастающим тогда и только тогда, когда функиия $а(\xi)$ удовлетворяет следующим условиям роста:

$$
c_{3}|\xi|^{p-1}-c_{4} \leqslant|a(\xi)| \leqslant c_{1}|\xi|^{p-1}+c_{2} \quad\left(c_{1}, c_{3}>0\right) .
$$


ДокАЗАТЕЛЬСтво. Правая часть в (1.2) совпадает с (1.1) и потому является необходимым и достаточным условием непрерьвности и ограниченности рассматриваемого оператора. Поэтому речь фактически идет о левой части неравенств (1.2). Сначала убедимся в достаточности выписанных условий для возрастания оператора $a$. Обозначим $X=\left(L^{p}(\Omega)\right)^{m}$ и $X^{\prime}=\left(L^{p^{\prime}}(\Omega)\right)^{m}$. Пусть задана последовательность функций $\varphi_{N}(x) \in X$, удовлетворяющих условию $\left\|\varphi_{N}\right\|_{X} \rightarrow \infty, N \rightarrow \infty$. Оценим норму функций $A \varphi_{N}$, используя левую часть соотношений (1.2):

$$
\left\|A \varphi_{N}\right\|_{X^{\prime}}^{p^{\prime}}=\int_{\Omega}\left|a\left(\varphi_{N}(x)\right)\right|^{p^{\prime}} d x \geqslant\left.\int_{\Omega}\left|c_{3}\right| \varphi_{N}(x)\right|^{p-1}-\left.c_{4}\right|^{p^{\prime}} d x .
$$

Для любых чисел $z \geqslant 0$ и $q>1$ очевидно неравенство

$$
\left|z-c_{4}\right|^{q} \geqslant c_{5} z^{q}-c_{6} \quad\left(c_{5}>0\right)
$$

и потому оценку для нормы функции $A \varphi_{N}$ можно продолжить следующим образом:

$$
\left\|A \varphi_{N}\right\|_{X^{\prime}}^{p^{\prime}} \geqslant \int_{\Omega}\left(c_{7}\left|\varphi_{N}(x)\right|^{p}-c_{6}\right) d x=c_{7}\left\|\varphi_{N}\right\|_{X}^{p}-c_{8} .
$$

Отсюда вытекает, что $\left\|A \varphi_{N}\right\|_{X^{\prime}} \rightarrow \infty$, если $\left\|\varphi_{N}\right\|_{X} \rightarrow \infty$, т.е. оператор $A$ при условиях (1.2) является возрастающим, что и утверждалось.

Перейдем к доказательству необходимости условий теоремы. Допустим, что оператор $A: X \rightarrow X^{\prime}$ является возрастающим, однако левое неравенство в (1.2) не вьполняется. Это значит, что найдется последовательность $\xi_{N} \in \mathbb{R}^{m}$ с такими свойствами:

$$
\left|\xi_{N}\right| \rightarrow \infty, \quad\left|a\left(\xi_{N}\right)\right|\left|\xi_{N}\right|^{-(p-1)} \rightarrow 0 \quad(N \rightarrow \infty)
$$

Построим последовательность функций $\varphi_{N} \in X$ так, чтобы вьполнялись следующие соотношения: $\left\|\varphi_{N}\right\|_{X} \rightarrow \infty$ при $N \rightarrow \infty$, однако $\left\|A \varphi_{N}\right\|_{X^{\prime}} \leqslant C$. Очевидно, это приводит к противоречию с условием возрастания оператора $A: X \rightarrow X^{\prime}$.

Ради некоторого упрощения записи предположим, что $a(0)=0$ и что область $\Omega \subset \mathbb{R}^{n}$ содержит нуль пространства $\mathbb{R}^{n}$. Тогда $\Omega$ содержит также шары $\omega_{N}=|x|<\rho_{N}$ при достаточно малых $\rho_{N} \rightarrow 0$. Последовательность точек $\xi_{N} \in \mathbb{R}^{m}$ из условия (1.3) считаем далее фиксированной. По ней строим последовательность функций, полагая $\varphi_{N}(x) \equiv \xi_{N}$, если $x \in \omega_{N}$, и считая $\varphi_{N}(x) \equiv 0$ вне $\omega_{N}$. Функции $A \varphi_{N}$ задаются тогда равенствами $a\left(\varphi_{N}(x)\right) \equiv a\left(\xi_{N}\right)$, если $x \in \omega_{N}$, и $a\left(\varphi_{N}(x)\right) \equiv 0$ вне $\omega_{N}$. Области $\omega_{N}$ укажем позднее. А пока вычислим нормы построенных функций:

$$
\begin{aligned}
\left\|\varphi_{N}\right\|_{X}^{p} & =\int_{\Omega}\left|\varphi_{N}(x)\right|^{p} d x=\int_{\omega_{N}}\left|\xi_{N}\right|^{p} d x=\left|\xi_{N}\right|^{p}\left|\omega_{N}\right| ; \\
\left\|A \varphi_{N}\right\|_{X^{\prime}}^{p^{\prime}} & =\int_{\omega_{N}}\left|a\left(\xi_{N}\right)\right|^{p^{\prime}} d x=\left|a\left(\xi_{N}\right)\right|^{p^{\prime}}\left|\omega_{N}\right| .
\end{aligned}
$$

Рассмотрим вначале основной вариант, считая, что $\left|a\left(\xi_{N}\right)\right| \rightarrow \infty$ при $N \rightarrow \infty$. Потребуем выполнения равенств $\left\|A \varphi_{N}\right\|_{X^{\prime}} \equiv 1$ для всех $N$. По формулам (1.5) однозначно определяются объемы шаров $\omega_{N}$ :

$$
\left|\omega_{N}\right|=\left|a\left(\xi_{N}\right)\right|^{-p^{\prime}} \quad(N=1,2, \ldots) .
$$


Отсюда очевидно, что $\left|\omega_{N}\right| \rightarrow 0$ при $N \rightarrow \infty$, так что шары $\omega_{N}$ неограниченно уменьшаются и при достаточно больших номерах $N \geqslant N_{0}$ войдут в заданную область $\Omega$. В результате построенные ранее функции $\varphi_{N}(x)$ для $N \geqslant N_{0}$ станут элементами пространства $X$, и при этом для них вьполняются соотношения $\left\|A \varphi_{N}\right\|_{X^{\prime}} \equiv 1\left(N \geqslant N_{0}\right)$. Подставляя формулы (1.6) в (1.4), находим, что для $N \geqslant N_{0}$

$$
\left\|\varphi_{N}\right\|_{X}^{p}=\left|\xi_{N}\right|^{p}\left|\omega_{N}\right|=\left|\xi_{N}\right|^{p}\left|a\left(\xi_{N}\right)\right|^{-p^{\prime}}=\left(\left|a\left(\xi_{N}\right)\right|\left|\xi_{N}\right|^{-(p-1)}\right)^{-p^{\prime}} .
$$

Вспоминая условие (1.3), приходим к выводу, что $\left\|\varphi_{N}\right\|_{X} \rightarrow \infty$ при $N \rightarrow \infty$. Одновременно $\left\|A \varphi_{N}\right\|_{X^{\prime}} \equiv 1$ для $N \geqslant N_{0}$. А это противоречит условию возрастания оператора $A: X \rightarrow X^{\prime}$. Аналогично рассматривается вариант, когда только некоторая подпоследовательность $\left|a\left(\xi_{N_{k}}\right)\right| \rightarrow \infty$ при $N_{k} \rightarrow \infty$. Остается изучить последний случай, который предполагает, что числа $\left|a\left(\xi_{N}\right)\right|$ равномерно ограничены. Тогда можно фиксировать некоторый шар $\omega_{0} \subset \Omega$ и считать $\omega_{N}=\omega_{0}$ для всех $N=1,2, \ldots$ Из равенств (1.4) вытекает, что $\left\|\varphi_{N}\right\|_{X} \rightarrow \infty$ при $N \rightarrow \infty$. В то же время по формулам $(1.5)\left\|A \varphi_{N}\right\|_{X^{\prime}} \leqslant C$ c единой постоянной $C$. Это противоречит условию возрастания оператора $A: X \rightarrow X^{\prime}$. Итак, предположив, что неравенства (1.2) для возрастающего оператора $A$ не вьполняются, мы пришли к противоречию, которое и доказывает справедливость теоремы.

Приведем пример монотонного возрастающего, но не коэрцитивного оператора суперпозиции.

ПримеР 1.1. Пусть $X=\left(L^{2}(\Omega)\right)^{2}$, т.е. множество вектор-функций $y(x)=\left(y_{1}(x)\right.$, $\left.y_{2}(x)\right)$, где $y_{1}, y_{2} \in L^{2}(\Omega)$. Очевидно, что $X$ - гильбертово пространство со скалярным произведением

$$
(y, z)=\int_{\Omega} y_{1}(x) z_{1}(x) d x+\int_{\Omega} y_{2}(x) z_{2}(x) d x \quad(y, z \in X) .
$$

В частности, $\|y\|_{X}^{2}=\left\|y_{1}\right\|_{L^{2}(\Omega)}^{2}+\left\|y_{2}\right\|_{L^{2}(\Omega)}^{2}$ и $X^{\prime}=X$.

Для $y=\left(y_{1}, y_{2}\right)$ полагаем $a y=\left(y_{2},-y_{1}\right)$. Определим оператор $A: X \rightarrow X^{\prime}$, считая $A y=a\left(y_{1}(x), y_{2}(x)\right)$ для $y \in X$. Нетрудно видеть, что $A$ - линейный оператор, определенный на всем пространстве $X$ и отображающий его в $X=X^{\prime}$, при этом

$$
\|A y\|_{X}^{2}=\int_{\Omega} y_{2}^{2}(x) d x+\int_{\Omega} y_{1}^{2}(x) d x=\|y\|_{X}^{2} .
$$

Из равенства (1.7) следует в частности, что $A$ - ограниченньй возрастающий оператор. Вьчислим еще скалярное произведение

$$
(A y, y)=\int_{\Omega} y_{2}(x) y_{1}(x) d x-\int_{\Omega} y_{1}(x) y_{2}(x) d x=0 .
$$

Полученное равенство показывает, что оператор $A$ не является коэрцитивным, однако остается монотонным, так как в силу линейности

$$
(A y-A z, y-z)=(A(y-z), y-z)=0 .
$$


2. Возрастающие дифференциальные операторы. В ограниченной области $\Omega \subset \mathbb{R}^{n}, n \geqslant 2$, с границей $\partial \Omega$ рассмотрим дифференциальное уравнение в дивергентной форме

$$
A u \equiv \sum_{|\alpha| \leqslant m}(-1)^{|\alpha|} D^{\alpha} A_{\alpha}\left(x, D^{\gamma} u\right)=f, \quad|\gamma| \leqslant m ;\left.\quad D^{\beta} u\right|_{\partial \Omega}=0, \quad|\beta| \leqslant m-1 .
$$

Здесь $\alpha=\left(\alpha_{1}, \ldots, \alpha_{n}\right), D^{\alpha}=D_{1}^{\alpha_{1}} \ldots D_{n}^{\alpha_{n}}, D_{i}=\partial / \partial x_{i}(i=1, \ldots, n)$.

Оператор $A$ удобно представлять как композицию трех операторов. Сначала задаем линейньй оператор $L u \equiv\left\{D^{\alpha} u ;|\alpha| \leqslant m\right\}$. Число компонент этого вектора обозначим через $r$. Оператор $L$ действует непрерывно из каждого соболевского пространства $X=\stackrel{\circ}{W}_{p}^{m}(\Omega), p>1$, в пространство $Y=\left(L^{p}(\Omega)\right)^{r}$. Затем вступает в действие нелинейный оператор суперпозиции $a(x, y): Y \rightarrow Y^{\prime}=\left(L^{p^{\prime}}(\Omega)\right)^{r}, p+p^{\prime}=p p^{\prime}$, который задается формулой

$$
a(x, y)=\left\{A_{\alpha}\left(x, y_{\gamma}(x)\right), \quad|\alpha| \leqslant m, \quad|\gamma| \leqslant m\right\} .
$$

Завершает все линейньй оператор $L^{\prime}: Y^{\prime} \rightarrow W_{p^{\prime}}^{-m}(\Omega)$, сопряженньй к $L$. В результатеполучаем композицию $A u=L^{\prime} a L u, u \in \stackrel{\circ}{W}_{p}^{m}(\Omega)$. Более детально указанная структура дифференциальных операторов рассматривается в [1, гл. II, §2]. Ясно, что нелинейность всего оператора $A$ определяется оператором суперпозиции $a: Y \rightarrow Y^{\prime}$, которьй в дальнейшем будем называть символом дифференциального оператора $A: \dot{\circ}_{p}^{m}(\Omega) \rightarrow$ $W_{p^{\prime}}^{-m}(\Omega)$.

Одна из задач теории дифференциальных операторов с частньми производными состоит в том, чтобы по свойствам символа $a: Y \rightarrow Y^{\prime}$ судить о свойствах дифференциального оператора $A$. Иногда это удается сделать достаточно просто. Например, если оператор $a(x, y): \mathbb{R}^{r} \rightarrow \mathbb{R}^{r}$ для каждого $x \in \mathbb{R}^{n}$ является монотонным, то дифференциальньй оператор $A$ также будет монотонным. Изучаемое здесь свойство возрастания оператора не является столь же просто наследуемым. Именно, если символ $a: Y \rightarrow Y^{\prime}$ является возрастающим, то это еще не гарантирует возрастания дифференциального оператора. Приведем соответствующий пример. В качестве символа возьмем оператор суперпозиции $a(y)$, построенньй в примере 1.1. Уже отмечалось, что $a(y)$ определяет линейньй ограниченный возрастающий и монотонный оператор из $\left(L^{2}(\Omega)\right)^{2}$ в себя. А теперь найдем соответствуюший дифференциальньй оператор. Он задается формулой

$$
A u \equiv \sum_{i=1}^{2} D_{i} A_{i}\left(D_{1} u, D_{2} u\right)=D_{1}\left(D_{2} u\right)+D_{2}\left(-D_{1} u\right) \equiv 0
$$

Как видим, возрастающий оператор суперпозиции $a(y)$ переводится композицией $L^{\prime} a L$ в нулевой оператор, которьй возрастающим быть не может. Это одна из причин, по которой при изучении дифференциальных операторов придерживаются условия строгой монотонности главной части оператора. Условие это имеет вид

$$
\sum_{|\alpha|=m}\left(A_{\alpha}\left(x, \xi_{0}, \xi^{m}\right)-A_{\alpha}\left(x, \xi_{0}, \eta^{m}\right)\right)\left(\xi_{\alpha}^{m}-\eta_{\alpha}^{m}\right)>0 \quad\left(\xi^{m} \neq \eta^{m}\right)
$$

Здесь $\left(\xi_{0}, \xi^{m}\right)=\xi$ и вектор $\xi^{m}$ соответствует производным $D^{\alpha} u$, у которых $|\alpha|=m$. 
Построенньй вьше пример показьвает, что условие возрастания дифференциального оператора $A: X \rightarrow X^{\prime}$ приходится устанавливать дополнительно к условию возрастания его символа $a: Y \rightarrow Y^{\prime}$. Для дифференциальных операторов часто удается установить еще одно свойство, которое напоминается далее. Говорят, что оператор $A: X \rightarrow X^{\prime}$ обладает $\left(S_{+}\right)$-свойством, если из соотношений $u_{N} \rightarrow u$ в $X, \overline{\lim }\left(A u_{N}, u_{N}-u\right) \leqslant 0$ $(N \rightarrow \infty)$ вытекает, что $u_{N} \rightarrow u$ в норме пространства $X$. Рассмотрим дифференциальньй оператор (2.1), сохранив в нем для наглядности только старшие производные. Другими словами, рассмотрим оператор

$$
A u \equiv \sum_{|\alpha|=m}(-1)^{m} D^{\alpha} A_{\alpha}\left(D^{m} u\right),\left.\quad D^{\beta} u\right|_{\partial \Omega}=0, \quad|\beta| \leqslant m-1 .
$$

Здесь $D^{m} u=\left\{D^{\alpha} u ;|\alpha|=m\right\}$. Число компонент этого вектора обозначим через $M$. Выпишем для оператора $A$ условия, обеспечивающие его возрастание и $\left(S_{+}\right)$-свойство:

$\left.\mathrm{A}_{1}\right)$ функции $A_{\alpha}(\xi)$ непрерывны по аргументу $\xi \in \mathbb{R}^{M}$;

$\mathrm{A}_{2}$ ) они удовлетворяют следующим условиям роста

$$
\left|A_{\alpha}(\xi)\right| \leqslant c_{1}|\xi|^{p-1}+c_{2}
$$

$\left.\mathrm{A}_{3}\right)$ для всех $\xi, \eta \in \mathbb{R}^{M}$

$$
\sum_{|\alpha|=m}\left(A_{\alpha}(\xi)-A_{\alpha}(\eta)\right)\left(\xi_{\alpha}-\eta_{\alpha}\right)>0 \quad(\xi \neq \eta)
$$

$\left.\mathrm{A}_{4}\right)$ для всех $\xi \in \mathbb{R}^{M}$

$$
\sum_{|\alpha|=m} A_{\alpha}(\xi) \xi_{\alpha} \geqslant c_{3}|\xi|^{p}-c_{4} \quad\left(c_{3}>0\right)
$$

Согласно теореме $2.1 \S 2$ гл. І монографии Скрыпника [4, с. 15] при условиях $\left.\mathrm{A}_{1}\right)-\mathrm{A}_{4}$ ) оператор $A: \stackrel{\circ}{W}_{p}^{m}(\Omega) \rightarrow W_{p^{\prime}}^{-m}(\Omega)$ является непрерьвньм, ограниченным и обладает $\left(S_{+}\right)$-свойством. Остается заметить, что условие $\left.\mathrm{A}_{4}\right)$ приводит к коэрцитивности дифференциального оператора $A$, откуда следует его возрастание. Таким образом, в приложениях к дифференциальным операторам для доказательства $\left(S_{+}\right)$-свойства привлекается условие коэрцитивности, которого старались избежать, введя понятие возрастающего оператора. Можно предположить, что условие $\mathrm{A}_{4}$ ) является излишним для доказательства возрастания оператора $A$ при сохранении остальных условий $\left.\mathrm{A}_{1}\right)-\mathrm{A}_{3}$ ). Следующее утверждение показьвает, что условие возрастания оператора $A$ необходимо приводит к условию $\mathrm{A}_{4}$ ), по крайней мере для однородных операторов, которые часто используются.

ТЕОРема 2.1. Пусть выполнены условия $\left.\left.\mathrm{A}_{1}\right)-\mathrm{A}_{3}\right)$, и пусть функции $A_{\alpha}(\xi)$ являются полохительно однородными, т.е. существует число $s>0$ такое, что $A_{\alpha}(\lambda \xi)=\lambda^{s} A_{\alpha}(\xi)$ для всех $\lambda>0 u|\alpha|=m$. При этих условиях оператор $A: \stackrel{\circ}{W}_{p}^{m}(\Omega) \rightarrow W_{p^{\prime}}^{-m}(\Omega)$ является возрастающим тогда и только тогда, когда выполнено также условие $\left.\mathrm{A}_{4}\right)$. В частности, оператор $A$ коэриитивен. 
ДокаЗАТЕЛЬСтво. Необходимость. Введем символ $a(\xi)=\left\{A_{\alpha}(\xi),|\alpha|=m\right\}$. Из условия теоремы вытекает, что символ является положительно однородной вектор-функцией: $a(\lambda \xi)=\lambda^{s} a(\xi), \lambda>0$. Отсюда получаем такое представление символа:

$$
a(\xi)=a(|\xi| \theta)=|\xi|^{s} a(\theta), \quad \theta=\xi|\xi|^{-1}, \quad|\theta|=1 .
$$

Переменная $\theta$ меняется в пределах единичной сферы $S_{M}$ пространства $\mathbb{R}^{M}$, которая является ограниченным множеством, и потому непрерывная функция $a(\theta)$ ограничена на $S_{M}$, так что $|a(\theta)| \leqslant C$ с фиксированной постоянной $C>0$. Отсюда и из представления (2.3) следует оценка

$$
|a(\xi)| \leqslant C|\xi|^{s} .
$$

Из условия ограниченности $\mathrm{A}_{2}$ ) сразу получаем, что $s \leqslant p-1$. Остается убедится, что $s=p-1$. Допустим, что $s<p-1$. Оценим норму функции $A u$. Полагая $X=\stackrel{\circ}{W}_{p}^{m}(\Omega)$, согласно определению нормы в сопряженном пространстве $X^{\prime}=W_{p^{\prime}}^{-m}(\Omega)$ имеем

$$
\|A u\|_{X^{\prime}}=\sup _{\|e\|_{X}=1}(A u, e)=\sup _{\|e\|_{X}=1} \int_{\Omega} \sum_{\alpha} A_{\alpha}\left(D^{m} u\right) D^{\alpha} e d x .
$$

Применив неравенство Гёльдера, получим

$$
\|A u\|_{X^{\prime}} \leqslant\left(\int_{\Omega}\left|a\left(D^{m} u\right)\right|^{p^{\prime}} d x\right)^{1 / p^{\prime}} \sup \|e\|_{W_{p}^{m}(\Omega)} .
$$

Последний множитель по условию есть единища, а к первому применим оценку (2.4), что дает

$$
\|A u\|_{X^{\prime}} \leqslant C\left(\int_{\Omega}\left|D^{m} u\right|^{q} d x\right)^{1 / p^{\prime}}=C\|u\|_{W_{q}^{m}(\Omega)}^{q / p^{\prime}} .
$$

Здесь $q=s p^{\prime}<p$, так как $s<p-1$. Хорошо известно, что пространство $W_{p}^{m}(\Omega)$ является собственным подмножеством пространства $\stackrel{\circ}{W}_{q}^{m}$ для $q<p$. Это значит, что найдется последовательность функций $u_{N} \in \stackrel{\circ}{W}_{p}^{m}(\Omega)$ с такими свойствами:

$$
\left\|u_{N}\right\|_{\mathscr{W}_{q}^{m}(\Omega)} \leqslant C, \quad\left\|u_{N}\right\|_{\mathscr{W}_{p}^{m}(\Omega)} \rightarrow \infty \quad(N \rightarrow \infty) .
$$

Используя неравенство (2.5), получаем

$$
\left\|A u_{N}\right\|_{X^{\prime}} \leqslant C\left\|u_{N}\right\|_{W_{q}^{m}(\Omega)}^{q / p^{\prime}} \leqslant C_{1},
$$

где $\left\|u_{N}\right\|_{X} \rightarrow \infty(N \rightarrow \infty)$. Это противоречит условию возрастания оператора $A$. Следовательно, строгое неравенство $s<p-1$ невозможно, так что $s=p-1$ и условие однородности принимает вид

$$
A_{\alpha}(\lambda \xi)=\lambda^{p-1} A_{\alpha}(\xi), \quad \lambda>0 .
$$

В частности, $A_{\alpha}(0)=0$ для всех $|\alpha|=m$. Введем функцию $H(\xi)=\sum_{|\alpha|=m} A_{\alpha}(\xi) \xi_{\alpha}$. Из условия $\left.\mathrm{A}_{3}\right)$ при $\eta=0$ вытекает, что $H(\xi)>0$ для $\xi \neq 0$. Пусть $\xi=|\xi| \theta$, где $|\theta|=1$. Тогда с учетом равенств (2.6) получаем

$$
H(\xi)=H(|\xi| \theta)=\sum_{|\alpha|=m} A_{\alpha}(|\xi| \theta)|\xi| \theta_{\alpha}=|\xi|^{p} \sum_{|\alpha|=m} A_{\alpha}(\theta) \theta_{\alpha} .
$$


Это значит, что

$$
H(\xi)=|\xi|^{p} H(\theta), \quad \theta=\xi|\xi|^{-1}, \quad|\theta|=1 .
$$

Так как переменная $\theta \in S_{M}$ принадлежит замкнутому ограниченному множеству, то функция $H(\theta)$ достигает на $S_{M}$ своего минимума $m_{0}$. По доказанному ранее $H(\xi)>0$ для $\xi \neq 0$. Следовательно, $m_{0}>0$. Таким образом, справедлива оценка $H(\theta) \geqslant m_{0}>0$, $|\theta|=1$. Из условия однородности $(2.7)$ получаем, что $H(\xi) \geqslant m_{0}|\xi|^{p}, m_{0}>0$. Вспоминая определение функции $H(\xi)$, перепишем последнее неравенство в виде

$$
\sum_{|\alpha|=m} A_{\alpha}(\xi) \xi_{\alpha} \geqslant m_{0}|\xi|^{p}, \quad m_{0}>0
$$

Это показывает, что для возрастающего оператора $A$ вьполнено условие $\left.\mathrm{A}_{4}\right)$, что и утверждалось.

Докажем достаточность условий $\left.\mathrm{A}_{1}\right)-\mathrm{A}_{4}$ ) для возрастания оператора $A$. Заметим, что эта часть доказательства не требует однородности функций $A_{\alpha}(\xi)$. Используя условие $\mathrm{A}_{4}$ ), оценим произведение

$$
(A u, u)=\int_{\Omega} \sum_{|\alpha|=m} A_{\alpha}\left(D^{m} u\right) D^{\alpha} u d x \geqslant \int_{\Omega}\left(c_{3}\left|D^{m} u\right|^{p}-c_{4}\right) d x=c_{3}\|u\|_{X}^{p}-c_{5} .
$$

Так как $|(A u, u)| \leqslant\|A u\|_{X^{\prime}}\|u\|_{X}$, то

$$
c_{3}\|u\|_{X}^{p}-c_{5} \leqslant\|A u\|_{X^{\prime}}\|u\|_{X}
$$

Отсюда очевидно, что $\|A u\|_{X^{\prime}} \rightarrow \infty$, если $\|u\|_{X} \rightarrow \infty$, что завершает доказательство теоремы.

Теорема 2.1 показьвает, что для дифференциальных операторов получить различие между возрастающими и коэрцитивными операторами довольно сложно.

Для однородных функций $A_{\alpha}(\xi)$ можно предложить следующий признак сравнения, несколько обобщающий условие коэрцитивности $\left.\mathrm{A}_{4}\right)$.

ТЕОРемА 2.2. Пусть выполнены условия $\left.\mathrm{A}_{1}\right)-\mathrm{A}_{3}$ ), а также

$$
\left.\mathrm{A}_{4}^{\prime}\right) \quad \sum_{|\alpha|=m} A_{\alpha}(\xi) \xi_{\alpha} \geqslant c_{3} a_{0}(\xi) \xi-c_{4} \quad\left(c_{3}>0\right)
$$

где вектор-функиия $a_{0}(\xi)$ положсительно однородна степени $s>0$, т.е. $a_{0}(\lambda \xi)=$ $\lambda^{s} a_{0}(\xi)$ для всех $\lambda>0$. Если дифференииальный оператор $A_{0}: \stackrel{\circ}{W}_{p}^{m}(\Omega) \rightarrow W_{p^{\prime}}^{-m}$, nостроенный по символу $a_{0}(\xi)$, является возрастающим, то оператор $A: \stackrel{\circ}{W}_{p}^{m}(\Omega) \rightarrow$ $W_{p^{\prime}}^{-m}$ также возрастает.

ДокАзАТЕльство. Так как оператор $A_{0}$ возрастает и его символ $a_{0}(\xi)$ есть положительно однородная функция, то по теореме 2.1 справедливо неравенство $a_{0}(\xi) \xi \geqslant m_{0}|\xi|^{p}$, $m_{0}>0$. В результате условие $\mathrm{A}_{4}^{\prime}$ ) можно представить в виде

$$
\sum_{|\alpha|=m} A_{\alpha}(\xi) \xi_{\alpha} \geqslant c_{3} m_{0}|\xi|^{p}-c_{2} \quad\left(c_{3}>0, \quad m_{0}>0\right)
$$

откуда следует возрастание оператора $A$, как это установлено при доказательстве достаточности в теореме 2.1 . 
3. Абстрактные возрастающие операторы. Далее часто будет использоваться следующее свойство. Пусть $\varphi(r)$ - непрерьвная строго монотонно возрастающая функция, определенная для $r \geqslant 0$ и удовлетворяющая условиям $\varphi(0)=0, \varphi(\infty)=\infty$.

ОПРЕДЕЛЕНИЕ 3.1. Будем говорить, что пространства $X, X^{\prime}$ обладают $J$-свойством, если существует оператор двойственности $J: X \rightarrow X^{\prime}$ относительно функции $\varphi(r)$, который является деминепрерьвным, ограниченным, монотонным и коэрцитивным.

Оператор двойственности тщательно изучен. Он определен для любой пары $X, X^{\prime}$ банаховых пространств, но может быть многозначным. Для его однозначности и деминепрерывности достаточно предполагать, что пространства $X, X^{\prime}$ строго вьпуклы. Оператор двойственности является монотонным и удовлетворяет следующим тождествам $[2$, гл. $2, \S 2]$ :

$$
(J u, u)=\|J u\|_{X^{\prime}}\|u\|_{X} ; \quad\|J u\|_{X^{\prime}}=\varphi\left(\|u\|_{X}\right) .
$$

Отсюда вытекает, в частности, его ограниченность и коэрцитивность. Напомним еще, что из деминепрерьвности следует радиальная непрерьвность. По аналогии с основной теоремой о монотонных коэрцитивных операторах сформулируем следующее утверждение.

ТЕОрема 3.1. Пусть пространства $X, X^{\prime}$ обладают J-свойством, и пусть оператор $A: X \rightarrow X^{\prime}$ является непрерывным, ограниченным, монотонным и возрастаюшим. Тогда уравнение $A u=f$ имеет решение $u \in X$ для любого әлемента $f \in X^{\prime}$.

ДокАЗАТЕЛЬСТво. Для каждого фиксированного числа $\varepsilon>0$ введем оператор $A_{\varepsilon}=$ $A+\varepsilon J$, где $J$ - оператор из определения 3.1. Отметим свойства нового оператора. Он радиально непрерьвен, ограничен и монотонен как сумма операторов с такими свойствами. Проверим, что он коэрцитивен. Из монотонности оператора $A$ следует неравенство $(A u, u) \geqslant(A(0), u)$, откуда $(A u, u) \geqslant-c_{1}\|u\|_{X}$, где $c_{1}=\|A(0)\|_{X^{\prime}}$. По условию оператор $J$ коэрцитивен, именно

$$
(J u, u)=\varphi\left(\|u\|_{X}\right)\|u\|_{X}, \quad \text { где } \varphi(r) \rightarrow \infty \quad(r \rightarrow \infty) .
$$

Из приведенных соотношений получаем

$$
\left(A_{\varepsilon} u, u\right) \geqslant-c_{1}\|u\|_{X}+\varepsilon \varphi\left(\|u\|_{X}\right)\|u\|_{X}=\left(\varepsilon \varphi\left(\|u\|_{X}\right)-c_{1}\right)\|u\|_{X} .
$$

Так как число $\varepsilon>0$ фиксировано и функция $\varphi(r) \rightarrow \infty$ при $r \rightarrow \infty$, то очевидно, что $\varepsilon \varphi(r)-c_{1} \rightarrow \infty$ при $r \rightarrow \infty$. Это означает коэрцитивность оператора $A_{\varepsilon}$. Подведем итог. Оператор $A_{\varepsilon}$ является радиально непрерьвным, ограниченньм, монотонным и коэрцитивным. Значит, для него вьполнены все условия основной теоремы о монотонных операторах $\left[1\right.$, с. 95], и потому уравнение $A_{\varepsilon} u^{\varepsilon}=f$ имеет решение $u^{\varepsilon} \in X$ для заданного элемента $f \in X^{\prime}$. Докажем возможность предельного перехода при $\varepsilon \rightarrow 0$, для чего выясним дополнительные свойства множества $u^{\varepsilon}, \varepsilon>0$. Уравнение $A_{\varepsilon} u^{\varepsilon}=f$, рассматриваемое как тождество в пространстве $X^{\prime}$, умножим на элемент $u^{\varepsilon} \in X$, что дает равенство $\left(A_{\varepsilon} u^{\varepsilon}, u^{\varepsilon}\right)=\left(f, u^{\varepsilon}\right)$. Его левую часть оценим снизу, используя соотношения (3.2). Правую часть оценим сверху через нормы. В результате получим неравенство

$$
\varepsilon \varphi\left(\left\|u^{\varepsilon}\right\|_{X}\right) \leqslant c_{2}, \quad c_{2}=c_{1}+\|f\|_{X^{\prime}}
$$


Подчеркнем, что постоянная $c_{2}$ не зависит от $\varepsilon$. Далее используем последнее из равенств (3.1). Получим

$$
\varepsilon\left\|J u^{\varepsilon}\right\|_{X^{\prime}}=\varepsilon \varphi\left(\left\|u^{\varepsilon}\right\|_{X}\right) \leqslant c_{2} .
$$

Уравнение $A_{\varepsilon} u^{\varepsilon}=f$ представим в виде $A u^{\varepsilon}=f-\varepsilon J u^{\varepsilon}$. Из оценки (3.3) вытекает, что

$$
\left\|A u^{\varepsilon}\right\|_{X^{\prime}} \leqslant\|f\|_{X^{\prime}}+\varepsilon\left\|J u^{\varepsilon}\right\|_{X^{\prime}} \leqslant\|f\|_{X^{\prime}}+c_{2} \equiv c_{3} .
$$

Убедимся в равномерной ограниченности множества $u^{\varepsilon}, \varepsilon>0$. Допустим, что найдется последовательность $u_{k}^{\varepsilon}, \varepsilon_{k} \rightarrow 0$, для которой $\left\|u^{\varepsilon_{k}}\right\|_{X} \rightarrow \infty$ при $\varepsilon_{k} \rightarrow 0$. Так как оператор $A$ по условию возрастающий, то $\left\|A u^{\varepsilon_{k}}\right\|_{X^{\prime}} \rightarrow \infty$, что противоречит оценке (3.4). Следовательно, найдется постоянная $c_{4}$ такая, что $\left\|u^{\varepsilon}\right\|_{X} \leqslant c_{4}$ для всех $\varepsilon>0$. Так как пространство $X$ рефлексивно, то можно выделить слабо сходящуюся последовательность $u^{\varepsilon_{m}} \rightarrow u$ в $X$ при $\varepsilon_{m} \rightarrow 0$. Оператор $J: X \rightarrow X^{\prime}$ ограничен, и потому из оценки $\left\|u^{\varepsilon}\right\|_{X} \leqslant c_{4}$ вытекает, что $\left\|J u^{\varepsilon}\right\|_{X^{\prime}} \leqslant c_{5}$, где постоянная не зависит от $\varepsilon$. Но тогда $\varepsilon J u^{\varepsilon} \rightarrow 0$ при $\varepsilon \rightarrow 0$ в пространстве $X^{\prime}$. Из тождества $A_{\varepsilon} u^{\varepsilon}=f$ следует в свою очередь, что $A u^{\varepsilon}=f-\varepsilon J u^{\varepsilon} \rightarrow f$ в $X^{\prime}$ при $\varepsilon \rightarrow 0$. Отсюда нетрудно получить, что $\left(A u^{\varepsilon_{m}}, u^{\varepsilon_{m}}\right) \rightarrow(f, u)$ при $\varepsilon_{m} \rightarrow 0$. Итак, построена последовательность $u^{\varepsilon_{m}}$ с такими свойствами:

$$
u^{\varepsilon_{m}} \rightarrow u \text { в } X, \quad A u^{\varepsilon_{m}} \rightarrow f \text { в } X^{\prime}, \quad \varlimsup_{\varepsilon_{m} \rightarrow 0}\left(A u^{\varepsilon_{m}}, u^{\varepsilon_{m}}\right) \leqslant(f, u) .
$$

Согласно [1, гл. III, $\S 1$, лемма 1.3, с. 85$]$ этих свойств в совокупности с монотонностью оператора $A$ достаточно, чтобы утверждать, что $A u=f$. Тем самым теорема доказана.

Заметим, что частный случай этой теоремы, когда $X$ - гильбертово пространство, доказан в монографии Ниренберга [7, гл. 5, п. 5.1, следствие 2].

Итак, требование коэрцитивности монотонного оператора удается ослабить до условия его возрастания. Условие монотонности также подвергалось многочисленным обобщениям. В результате появились, например, псевдомонотонные операторы, операторы вариационного исчисления, операторы с $(S)$-свойством и другие. Приходится констатировать, что в приложениях к уравнениям с частными производными у перечисленных классов не оказалось преимуществ перед ранее введенным множеством операторов, обладающих $(M)$-свойством. Так называются операторы, для которых равенство $A u=f$ вытекает из следующих соотношений, выполняющихся для последовательности $u_{N} \in X$ при $N \rightarrow \infty:$

$$
u_{N} \rightarrow u \text { в } X, \quad A u_{N} \rightarrow f \text { в } X^{\prime}, \quad \varlimsup \lim \left(A u_{N}, u_{N}\right) \leqslant(f, u) .
$$

Согласно [2, замечание 2.1, гл. 2, § 2] в этом определении аксиоматизируется предельный переход в галёркинских приближениях. Класс операторов, обладающих $(M)$-свойством, довольно широк. Например, любой линейньй ограниченньй оператор обладает $(M)$-свойством в силу слабой непрерьвности.

Напомним следующее утверждение [2, гл. 2, §2, теорема 2.1]. Пусть оператор $A$ : $X \rightarrow X^{\prime}$ является деминепрерьвньп, ограниченньг, коэрцитивньп и обладает $(M)$ свойством. Тогда уравнение $A u=f$ имеет решение $u \in X$ для каждого элемента $f \in X^{\prime}$. Приведем параллельные утверждения для нечетных операторов, т.е. операторов, удовлетворяющих условию $A(-u)=-A u$. Подобные утверждения установлены в работе [8]. Здесь они приводятся по монографии [4, гл. 1, § 5, следствие 5.1 и теорема 5.2]. 
ПРЕДЛОЖЕНИЕ 3.1. Пусть $A: X \rightarrow X^{\prime}$ является непрерывным, ограниченным, возрастающим нечетным оператором, и пусть он обладает $\left(S_{+}\right)$-свойством. Тогда уравнение $A u=f$ имеет решение $u \in X$ для кажсдого әлемента $f \in X^{\prime}$.

ПРЕДЛОЖЕНИЕ 3.2. Пусть $A: X \rightarrow X^{\prime}$ является непрерывным, ограниченным, нечетным, положительно однородным оператором, причем уравнение $A и=0$ допускает единственное решение $u=0$. Пусть еще оператор $A$ обладает $\left(S_{+}\right)$-свойством. Тогда уравнение $A u=f$ имеет решение $u \in X$ для кажсдого элемента $f \in X^{\prime}$.

В предложении $3.1\left(S_{+}\right)$-свойство можно заменить на монотонность оператора $A$ и $J$-свойство, как это следует из теоремы 3.1. Убедимся на примере, что ослабить монотонность до $(M)$-свойства уже нельзя при сохранении остальных условий предложения 3.1 .

ПРИмеР 3.1. Пусть $H$ - сепарабельное гильбертово пространство с ортонормированньп базисом $\left\{e_{k}\right\}_{k=1}^{\infty}$. Введем линейньй оператор поворота, которьй на базисных векторах действует по закону $A e_{k}=e_{k+1}(k=1,2, \ldots)$. Если $x=\sum_{k=1}^{\infty} c_{k} e_{k}$, то полагаем $A x=\sum_{k=1}^{\infty} c_{k} e_{k+1}$. Очевидно, что $\|A x\|=\|x\|$ для всех $x \in H$ и потому оператор $A$ - линейный, ограниченный, непрерьвньй и возрастающий из пространства $H$ в себя, причем $A(-x)=-A x$, т.е. это нечетньй оператор.

Убедимся, что он обладает $(M)$-свойством. Действительно, если $x_{n} \rightarrow x, A x_{N} \rightarrow f$ в $H$ при $N \rightarrow \infty$, то $A x=f$, так как линейньй ограниченный оператор слабо непрерывен. Равенство $A x=f$, полученное из слабых сходимостей, характеризует операторы c $(M)$-свойством. Итак, построенньй оператор $A$ обладает всеми свойствами оператора из предложения 3.1, кроме $\left(S_{+}\right)$-свойства, которое заменено на $(M)$-свойство. Тем не менее, уравнение $A x=f$ не имеет решения для $f=e_{1}$, т.е. заключение предложения 3.1 для введенного оператора $A$ несправедливо.

Убедимся далее, что в предложении $3.2\left(S_{+}\right)$-свойство нельзя заменить даже на монотонность.

ПРИмеР 3.2. Пусть снова $H$ - сепарабельное гильбертово пространство, и пусть $A$ : $H \rightarrow H$ - линейньй, ограниченньй, положительньй, вполне непрерывный оператор. Такой оператор положительно однороден степени $k=1$ и нечетен в силу линейности, так как $A(t x)=t A x(t>0), A(-x)=-A x$. Он также является монотонньм в силу положительности: $(A x, x)>0, x \neq 0$. Уравнение $A x=0$ имеет единственное решение $x=0$ также в силу положительности оператора $A$. Тем неменее, уравнение $A x=f$ допускает решения не для любой правой части $f \in H$, что подтверждает невозможность ослабления $\left(S_{+}\right)$-свойства оператора $A$ до его монотонности.

Теорема 3.1 показывает, что возрастание оператора $A$ в совокупности с монотонностью достаточно для существования решения уравнения $A u=f$. Ослабить условие монотонности до $(M)$-свойства без дополнительньх предположений уже не удается, как показывает пример 3.1. В следующем утверждении предложены дополнительные условия, которые дают возможность изучать возрастающие операторы с $(M)$-свойством.

ТЕОрема 3.2. Пусть пространства $X, X^{\prime}$ обладают J-свойством, и пусть оператор $A: X \rightarrow X^{\prime}$ является непрерывным, ограниченным, возрастающим и обладает (M)-свойством. Пусть еще для него выполнены следующие два условия:

1) форма $(A u, u)$ полуограничена снизу; именно $(A u, u) \geqslant 0$ для всех $u \in X$; 
2) если последовательность $u_{N} \rightarrow$ и в $X$ при $N \rightarrow \infty$, то

$$
\liminf _{N \rightarrow \infty}\left(A u_{N}, u_{N}-u\right) \geqslant 0 \text {. }
$$

Тогда уравнение $A u=f$ имеет решение $u \in X$ для любого әлемента $f \in X^{\prime}$.

ДоказАтЕльСтво. Фиксируем число $\varepsilon$ и введем оператор $A_{\varepsilon}=A+\varepsilon J$. Он деминепрерьвен и ограничен, а также коэрцитивен, что устанавливается аналогично тому, как это сделано при доказательстве предыдущей теоремы 3.1. Убедимся еще, что оператор $A_{\varepsilon}$ обладает $(M)$-свойством. Пусть задана последовательность $u_{N} \in X$, для которой известны такие соотношения при $N \rightarrow \infty$ :

$$
u_{N} \rightarrow u \text { в } X, \quad \varlimsup\left(A_{\varepsilon} u_{N}, u_{N}-u\right) \leqslant 0 .
$$

Последнее неравенство запишем подробнее:

$$
\varlimsup_{N \rightarrow \infty}\left[\left(A u_{N}, u_{N}-u\right)+\varepsilon\left(J u_{N}, u_{N}-u\right)\right] \leqslant 0 .
$$

Для участвующего здесь оператора двойственности установим одно свойство.

Лемма 3.1. Пусть $u_{N} \rightarrow$ в $X$ при $N \rightarrow \infty$. Тогда $\liminf _{N \rightarrow \infty}\left(J u_{N}, u_{N}-u\right) \geqslant 0$.

ДокАЗАТЕЛЬСТво. Действительно, оператор двойственности является монотонным, и потому вьполняется неравенство $\left(J u_{N}-J u, u_{N}-u\right) \geqslant 0$, которое представим в виде

$$
\left(J u_{N}, u_{N}-u\right) \geqslant\left(J u, u_{N}-u\right) .
$$

Правая часть здесь стремится к нулю при $N \rightarrow \infty$, откуда и вытекает утверждение леммы.

Доказательство следующей леммы достаточно очевидно.

Лемма 3.2. Пусть последовательности чисел $a_{N}, b_{N}$ ограничены и при $N \rightarrow \infty$ удовлетворяют соотношениям

$$
\varlimsup\left(a_{N}+b_{N}\right) \leqslant 0, \quad \liminf a_{N} \geqslant 0, \quad \liminf b_{N} \geqslant 0 .
$$

Тогда существуют предель $\lim a_{N}=0, \lim b_{N}=0$.

Вернемся к неравенству (3.6) и введем обозначения

$$
a_{N}=\left(A u_{N}, u_{N}-u\right), \quad b_{N}=\varepsilon\left(J u_{N}, u_{N}-u\right) .
$$

Проверим вьполнение условий леммы 3.2. Ее первое неравенство совпадает с (3.6), второе входит в условие теоремы, а последнее вытекает из леммы 3.1. Следовательно, существуют $\lim _{N \rightarrow \infty} a_{N}=0$ и $\lim _{N \rightarrow \infty} b_{N}=0$, что при возвращении к исходньм обозначениям дает

$$
\lim _{N \rightarrow \infty}\left(A u_{N}, u_{N}-u\right)=0, \quad \lim _{N \rightarrow \infty} \varepsilon\left(J u_{N}, u_{N}-u\right)=0 .
$$


Отсюда следует, что $A u_{N} \rightarrow A u, J u_{N} \rightarrow J u$ при $N \rightarrow \infty$, так как операторы $A$ и $J$ обладают $(M)$-свойством, и соответственно $A_{\varepsilon} u_{N} \rightarrow A_{\varepsilon} u(N \rightarrow \infty)$. Остается напомнить, что последняя сходимость получена в предположении, что выполняются соотношения (3.5). Согласно определению это значит, что оператор $A_{\varepsilon}$ обладает $(M)$-свойством. Подведем итог. Оператор $A_{\varepsilon}$ является деминепрерьвным, ограниченньм, коэрцитивным и обладает $(M)$-свойством. Этого достаточно, чтобы утверждать, что уравнение $A_{\varepsilon} u^{\varepsilon}=f$ имеет решение $u^{\varepsilon} \in X$ для заданного элемента $f \in X^{\prime}$.

Итак, для каждого $\varepsilon>0$ определена функция $u^{\varepsilon}$, удовлетворяющая уравнению

$$
A u^{\varepsilon}+\varepsilon J u^{\varepsilon}=f
$$

Остается убедится, что возможен предельньй переход при $\varepsilon \rightarrow 0$. Заметим, что эта часть доказательства аналогична предельному переходу в предыдущей теореме, и потому отметим только основные моменты. Уравнение (3.7) умножим на $u^{\varepsilon}$. Используя оценки снизу для форм $(A u, u)$ и $(J u, u)$, получаем неравенство $\varepsilon \varphi\left(\left\|u^{\varepsilon}\right\|_{X}\right) \leqslant c_{2}$. Отсюда аналогично (3.3) получаем оценку $\varepsilon\left\|J u^{\varepsilon}\right\|_{X^{\prime}} \leqslant c_{2}$. Снова обращаясь к уравнению (3.7), по аналогии с (3.4) вьводим, что $\left\|A u^{\varepsilon}\right\|_{X^{\prime}} \leqslant c_{3}$. Так как оператор $A$ по условию возрастающий, то отсюда следует равномерная оценка $\left\|u^{\varepsilon}\right\|_{X} \leqslant c_{4}$, которая дает неравенство $\left\|J u^{\varepsilon}\right\|_{X^{\prime}} \leqslant c_{5}$ и сходимость $\varepsilon J u^{\varepsilon} \rightarrow 0$ в $X^{\prime}$ при $\varepsilon \rightarrow 0$. В результате, из уравнения $(3.7)$ вытекает, что $A u^{\varepsilon} \rightarrow f$ в $X^{\prime}$ при $\varepsilon \rightarrow 0$. Изложенное означает, что можно выбрать последовательность $u^{\varepsilon_{k}}, \varepsilon_{k} \rightarrow 0$, с такими свойствами:

$$
u^{\varepsilon_{k}} \rightarrow u \text { в } X, \quad A u^{\varepsilon_{k}} \rightarrow f \text { в } X^{\prime}, \quad \lim \left(A u^{\varepsilon_{k}}, u^{\varepsilon_{k}}\right)=(f, u) .
$$

Оператор $A$ обладает $(M)$-свойством, которое предполагает, что соотношений (3.8) достаточно для равенства $A u=f$. Это завершает доказательство теоремы.

В заключение автор благодарит Ю.А. Дубинского и С.И. Похожаева за полезное обсуждение результатов.

\section{СПИСОК ЦИТИРОВАННОЙ ЛИТЕРАТУРЫ}

[1] Гаевский X., Грегер К., Захариас К. Нелинейные операторные уравнения и операторные дифференциальные уравнения. М.: Наука, 1978.

[2] Лионс ЖК.-Л. Некоторые методы решения нелинейных краевых задач. М.: Мир, 1972.

[3] Дубинский Ю. А. Нелинейные эллиптические и параболические уравнения // Современные проблемы математики. Т. 9. М.: ВИНИТИ, 1976. С. 5-130.

[4] Скрыпник И. В. Методы исследования нелинейных эллиптических граничных задач. М.: Наука, 1990.

[5] Милоевич П. С. Разрешимость сильно нелинейных операторных уравнений и их приложения // Дифференц. уравнения. 1995. Т. 31. № 3. С. 502-516.

[6] Красносельский М. А. Топологические методы в теории нелинейных интегральных уравнений. М.: Гостехиздат, 1956.

[7] Ниренберг Л. Лекции по нелинейному функциональному анализу. М.: Мир, 1977.

[8] Похожаев С.И. О разрешимости нелинейных уравнений с нечетными операторами // Функцион. анализ и его прилож. 1967. Т. 1. №3. С. 66-73. 